\title{
Coeus: Consistent and Continuous Network Update in Software-Defined Networks
}

\author{
Xin $\mathrm{He}^{\dagger}$, Jiaqi Zheng ${ }^{\dagger}$, Haipeng Dai ${ }^{\dagger}$, Chong Zhang ${ }^{\dagger}$, Wajid Rafique ${ }^{\dagger}$, Geng $\mathrm{Li}^{\ddagger}$, Wanchun $\mathrm{Dou}^{\dagger}$, Qiang $\mathrm{Ni}^{\sharp}$, \\ ${ }^{\dagger}$ State Key Laboratory for Novel Software Technology, Nanjing University, China \\ $\ddagger$ Department of Computer Science, Yale University, USA \\ $\sharp$ School of Computing and Communications, Lancaster University, UK
}

\begin{abstract}
Network update enables Software-Defined Networks (SDNs) to optimize the data plane performance via southbound APIs. The single update between the initial and the final network states fail to handle high-frequency changes or the burst event during the update procedure in time, leading to prolonged update time and inefficiency. On the contrary, the continuous update can respond to the network condition changes at all times. However, existing work, especially "Update Algebra" can only guarantee blackhole- and loop-free. The congestion-free property cannot be respected during the update procedure. In this paper, we propose Coeus, a continuous network update system while maintaining blackhole-, loop- and congestion-free simultaneously. Firstly, we establish an operation-based continuous update model. Based on this model, we dynamically reconstruct an operation dependency graph to capture unexecuted update operations and the link utilization variations. Subsequently, we develop an operation composition algorithm to eliminate redundant update commands and an operation node partition algorithm to speed up the update procedure. We prove that the partition algorithm is optimal and can guarantee the consistency. Finally, extensive evaluations show that Coeus can improve the makespan by at least $179 \%$ compared with state-of-the-art approaches when the arrival rate of update events equals to three times per second.
\end{abstract}

\section{INTRODUCTION}

Software-Defined Networks (SDNs) outsource the network control function over switches to the logically-centralized controller. Benefitting from the global view of the controller and the simplified data plane, SDNs can provide flexible traffic management and fine-grained network monitoring (e.g., traffic engineering [1], [13], [14], [34] and failures recovery [7], [29], [33]). Due to high-frequency network condition variations, the controller requires handling continuous update events rapidly to optimize the performance of the data plane [5], [27]. Therefore, the network needs to have the ability to perform update events as soon as possible and guarantee consistency [2], [6], [31] (i.e., blackhole-, loop- and congestion-free) during updates.

Given the initial and the final network states in the data plane, the single update [4], [9], [18], [23], [25], [26], [32] advocates determining a consistent update sequence to shorten the update time. However, they fail to handle high-frequency changes or the burst event during the update procedure. Each update event can only be executed after the completion of the previous update events, leading to prolonged update time and poor performance. Instead of the single update, existing work, especially "Update Algebra" [11] explores the continuous update solution to respond to network condition variations, where the update events arrive in an online manner. "Update Algebra" models operations in continuous update events as a set of projections and selects a feasible subset of projections to execute.

Although "Update Algebra" [11] has the ability to handle continuous update events in an online manner, it can only guarantee blackhole- and loop-free properties. Essentially, "Update Algebra" mainly considers the order of update operations in the flow-based granularity, but different flows compete for the limited link bandwidth resource. The transient congestion may occur during updates, which incurs packets loss and degrades network performance.

In this paper, we initiate the study of consistent continuous network update problem, which can handle the frequent update events in an online manner and guarantee blackhole-, loopand congestion-free properties simultaneously. To the best of our knowledge, little is known today about how to schedule flows in this problem. Performing the consistent continuous update remains algorithmically challenging. We face three main challenges: (i) Performing continuous updates requires the controller to identify which update commands have been executed, which ones are being executed, and which ones will be executed. Besides, the time-varying network resources and update commands should be jointly considered to produce a consistent update sequence. (ii) The number of possible congestion-free update orders is exponential many even for a single update event [15]. For an update event containing $n$ flows which need to be rerouted, the number of possible update orders is $O(n !)$. Involving multiple update events makes the problem essentially harder. (iii) We need to speed up the continuous update procedure, i.e., increasing the degree of parallelism. We wish to find as many independent parts as possible to execute them simultaneously in a consistent way.

To address the challenges mentioned above, we make the following contributions. Firstly, we develop Coeus, a continuous network update system to maintain consistency. We give an overview of Coeus (Sec. III). Besides, we extend the continuous model proposed in [11], which only captures the data plane update. We establish the operation-based continuous update model, which can capture both the update event in the control plane and forwarding actions in the data plane (Sec. IV). Secondly, we propose a set of algorithms to achieve the consistent continuous update (Sec. V). Specifically, based on the continuous update model, we dynamically reconstruct 

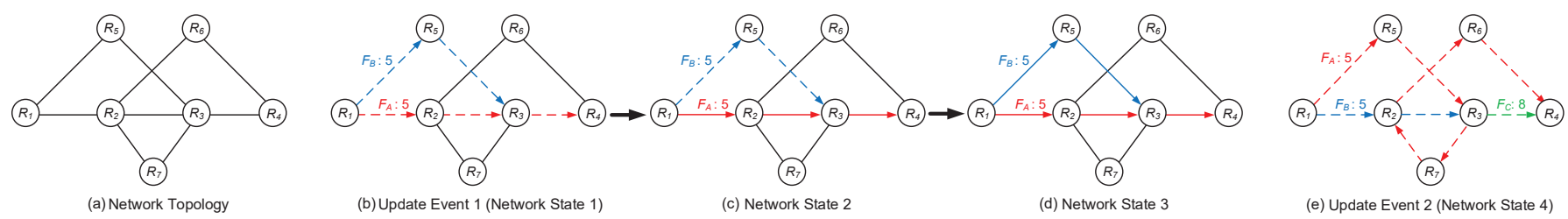

Fig. 1. A continuous update example. Fig. 1(a) shows the network topology. Fig. 1(b) Fig. 1(e) denote the update events and network states.

an operation dependency graph to capture utilization variations of each link and dependencies of unexecuted update operations in continuous update events. However, an update operation from the newly arrived event may cancel out an existing unexecuted update operation. We develop the operation composition algorithm in the dependency graph to eliminate redundant operations. By leveraging the dynamic operation dependency graph and operation composition, we solve the first two challenges. To address the third challenge, we design the operation node partition algorithm to split the operation node into a series of independent suboperation nodes. The update of independent suboperation nodes improves the degree of update parallelism since parts of flow path can be updated directly, and the flow does not need to wait until all link resources along its target path are sufficient. We prove that our node partition algorithm is optimal, and the update of suboperation nodes ensures the consistency. Finally, we evaluate Coeus by using experiments in large-scale simulations (Sec. VI). We use two common topologies (i.e., SWAN and fat-tree) to verify the effectiveness of Coeus by changing the arrival rate of update events constantly. The simulations show that Coeus improves the makespan by at least $179 \%$ compared with state-of-the-art approaches when the arrival rate of update events equals to three times per second.

\section{A Motivating Example}

In this section, we use a motivating example to illustrate the continuous update problem. For convenience, we summarize important notions in the Table I.

Fig. 1(a) shows a network topology containing of seven switches $\left\{R_{1}, \ldots, R_{7}\right\}$, where the capacity of link $\left\langle R_{1}, R_{5}\right\rangle$ equals to 5 units and others equal to 10 units. Two update events $U E_{1}$ and $U E_{2}$ arrive sequentially. The update event $U E_{1}$ will install forwarding rules of two flows $F_{A}$ and $F_{B}$, where demands of $F_{A}$ and $F_{B}$ are both 5 units. The update event $U E_{2}$ will install forwarding rules of flow $F_{C}$, where the demand of $F_{C}$ is 8 unit. Each update event incurs different network states, and each network state captures different network routing information. We use the directed edge to denote the routing of each flow. The dashed line characterizes that the forwarding rules of the flow have not been installed while the solid line characterizes that the forwarding rules of the flow have already been installed. Fig. 1(b) denotes that when the update event $U E_{1}$ appears, the controller will assign the path of flows $F_{A}$ and $F_{B}$. However, the corresponding forwarding commands have not been installed in switches. Fig. 1(c) shows that in the network state $N S_{2}, F_{A}$ has been updated to its target path while $F_{B}$ has not been updated. The network state $N S_{3}$ shown in Fig. 1(d) indicates that $F_{B}$ has been updated, and the update event $U E_{1}$ is finished since all of the flows in $U E_{1}$ are routed through their target paths. Fig. 1(e) shows that when $U E_{2}$ occurs after $U E_{1}$, the controller will assign the route of flow $F_{C}$ and change the routes of $F_{A}$ and $F_{B}$ due to the limited capacity of link $\left\langle R_{3}, R_{4}\right\rangle$.

TABLE I

KEY NOTATIONS

\begin{tabular}{c|l}
\hline Notation & Meaning \\
\hline$G$ & Acyclic directed network graph $G=(V, E)$ \\
$V$ & Set of switches $\{v\}$ \\
$E$ & Set of links $\{\langle u, v\rangle\}$ \\
$c_{u, v}$ & Capacity of link $\langle u, v\rangle$ \\
$F$ & Set of flows $\left\{f_{i}\right\}$ \\
$d^{f_{i}}$ & Demand of flow $f_{i}$ \\
$U E$ & Update event \\
$\widehat{U E}$ & Set of unexecuted update operations in $U E$. \\
$N S$ & Network state \\
$N S_{i n i t}$ & Relative initial state \\
$O_{I}$ & Set of executed update operations \\
$O_{N I}$ & Set of unexecuted update operations \\
$O^{f_{i}}$ & Set of unexecuted update operations of $f_{i}$ \\
$o_{i}$ & Update operation of $f_{i}$ \\
$R_{u, v}$ & Resource nodes in the operation dependency graph \\
$r_{u, v}$ & Residual resource of the resource node \\
\hline
\end{tabular}

In the single update scheme, the update event must wait for the completion of previous update events, e.g., if $U E_{2}$ occurs after $N S_{1}, U E_{2}$ cannot be responded until $U E_{1}$ is finished. This serial update scheme prolongs the makespan significantly. We aim to develop a system to respond random and continuous update events. However, dealing with the continuous update in an online manner and guaranteeing blackhole-, loop- and congestion-free simultaneously are full of challenges.

For example, under the condition where $U E_{1}$ has installed the forwarding rule of $F_{A}$ on the switch $R_{2}$ and has not installed the forwarding rule of $F_{A}$ on the switch $R_{3}, U E_{2}$ occurs. Then, the update event $U E_{1}$ encourages $R_{3}$ to install the following forwarding rule for $F_{A}$ (i.e., forwarding $F_{A}$ to switch $R_{4}$ ) while the update event $U E_{2}$ encourages $R_{3}$ to install another forwarding rule for $F_{A}$ (i.e., forwarding $F_{A}$ to switch $R_{7}$ ). Therefore, handling continuous update events simply may lead to chaotic routing. Besides, switches must have forwarding rules of each incoming flow, and the flow should not have transient loops during updates (i.e., maintaining blackhole- and loop-free). For example, when $U E_{2}$ occurs after $N S_{2}$, the switch $R_{5}$ should has the forwarding rule of $F_{A}$ before $F_{A}$ routes through the link $\left\langle R_{1}, R_{5}\right\rangle$. Besides, the forwarding rules of $F_{A}$ should be installed on switch $R_{3}$ and $R_{7}$ after the original forwarding rule on switch 


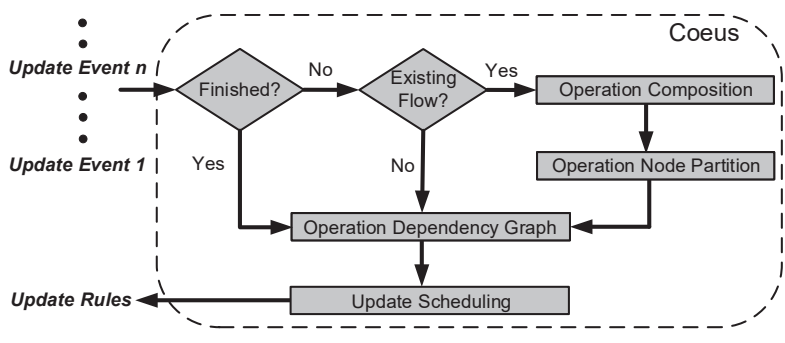

Fig. 2. Overview of Coeus.

$R_{2}$ is removed. Otherwise, the transient loop $\left\langle R_{2}, R_{3}\right\rangle \rightarrow$ $\left\langle R_{3}, R_{7}\right\rangle \rightarrow\left\langle R_{7}, R_{2}\right\rangle$ appears. To guarantee a congestionfree condition, the update order of multiple flows should be carefully calculated. For example, when $U E_{2}$ occurs after $N S_{3}, F_{A}$ should not be updated before $F_{B}$. Otherwise, link congestion will occur due to the limited link resource of $\left\langle R_{1}, R_{5}\right\rangle$. Similarly, $F_{C}$ should be updated after $F_{A}$. To speed up the continuous update procedure, the concurrency of update should be taken into consideration. We will introduce the corresponding method in detail in Sec. V-B.

\section{Coeus OVERVIEW}

To solve the problems mentioned above, we propose a system, Coeus, to achieve the consistent and continuous update. In this section, we will introduce an overview of Coeus.

The entire workflow of Coeus is shown in Fig. 2. In SDNs, the update events come in an online manner. To handle continuous updates, the centralized controller firstly judges whether the previous update events are finished. Once the condition is true, we build the operation dependency graph which has a set of resource nodes and a set of operation nodes to capture the link utilization variations and the dependencies of update operations. Otherwise, we make further judgment about whether updated flows which involved in the new update event have already existed in the data plane. If flows do not have forwarding paths at this moment (e.g., $F_{C}$ in Fig. 1(e)), we insert new operation nodes of flows in the operation dependency graph and construct corresponding relationships. Otherwise, update operations are added into existing operation nodes, and we dynamically reconstruct the dependency relationship in the dependency graph. However, adding update operations into operation nodes continuously may lead to redundant operations in operation nodes. By compositing update operations, Coeus replaces redundant operations into equivalent ones. Subsequently, Coeus designs the operation node partition algorithm to increase the parallelism of updates and speed up the continuous update procedure. The node partition algorithm divides the operation node into several suboperation nodes which can be updated independently in a blackhole- and loop-free manner. Finally, by leveraging the dynamic dependency graph, our update scheduling scheme sends a set of update commands to the data plane.

\section{Continuous Updates Model}

After describing Coeus, we will introduce the continuous update model in this section. The general network can be modeled as a graph $G=(V, E)$, where $V$ is the set of switches and $E \subseteq V^{2}$ is the set of links. Each link $\langle u, v\rangle \in E$ has a capacity $c_{u, v} . F$ is the set of $s-d$ flows in the network, where $s$ and $d$ are the source and destination of flow $f$. Each flow $f_{i} \in F$ is an unsplittable flow with demand $d^{f_{i}}$.

In the control plane, continuous update events $\{U E\}$ arrive randomly. Each update event $U E=\left\{o^{f_{i}} \mid \forall f_{i} \subseteq F\right\}$ contains a set of update operations $\left\{o^{f_{i}}\right\}$ to assign the route of each flow $f_{i}$. We define $\widehat{U E} \in U E$ to characterize the set of operations that have not been executed in the data plane. The network states $\{N S\}$ present the current route information captured by the control plane, where $N S=\left\{O_{I}, O_{N I}\right\}$. $O_{I}$ denotes the set of operations that have been executed while $O_{N I}$ denotes the set of operations that have not been executed in the data plane. When the $n$-th update event arises, $O_{I}$ and $O_{N I}$ can be expressed as follows.

$$
\begin{gathered}
O_{I}=\left\{\left(U E_{1}-\widehat{U E_{1}}\right) \cup\left(U E_{2}-\widehat{U E_{2}}\right) \ldots \cup\left(U E_{n}-\widehat{U E_{n}}\right)\right\} \\
O_{N I}=\left\{\widehat{U E_{1}} \cup \widehat{U E_{2}} \ldots \cup \widehat{U E_{n}}\right\}
\end{gathered}
$$

In this paper, we focus on scheduling unexecuted update operations orderly. We introduce $O^{f_{i}}$ to denote the set of unexecuted operations of $f_{i}$, i.e., $O^{f_{i}}=O_{N I}^{f_{i}}$. Different from the single update with the deterministic initial and final state, continuous update events with unknown update operations make it hard to determine the final state of the network. We define the relative initial state as follows.

Definition IV.1. Relative Initial State: The relative initial state represents that all operations of the previous update events $\{U E\}$ have been executed in the data plane, i.e., all of the previous update events $\{U E\}$ are completed.

Now we discuss the mapping relationship between update operations in the control plane and forwarding actions in the data plane. The controller sends update operations to individual switches. Once an update operation $o^{f_{i}}$ is executed on the specified switch, it will execute the corresponding action if $f_{i}$ matches the flow entry in the switch flow table. We classify update operations into three types $\{a d d, \bmod , d e l\}$. At the specified switch $u$, once the add operation $o_{a d d}^{f_{i}}$ : $a d d\left\{f w d \_v\right\}$ is executed, the forwarding rule of $f_{i}$ is added, and $f_{i}$ will be forwarded to switch $v$. Once the del operation $o_{d e l}^{f_{i}}:$ del $\left\{f w d \_v\right\}$ is executed, the forwarding rules of $f_{i}$ is deleted from the switch. Similarly, the mod operation $o_{\text {mod }}^{f_{i}}: \bmod \left\{f w d \_v \rightarrow f w d \_w\right\}$ denotes that modifying the forwarding rules of $f_{i}$ enables $f_{i}$ to be forwarded to switch $w$ instead of $v$. Indeed, $o_{\text {mod }}^{f_{i}}$ can be treated as a delete operation $o_{d e l}^{f_{i}}$ that removes the original forwarding rule, and an add operation $o_{a d d}^{f_{i}}$ that adds the new forwarding rule. In the continuous update, not all of the update operations are valid, we define the validity of update operations as follows.

Definition IV.2. Valid Update Operations: The valid update operations of flow depend on the previous operations of the same flow acting on the same switches, valid update operations enable each switch to have at most one forwarding rule for each flow, and the repeated forwarding rule is forbidden. 
TABLE II

VALID UPDATE OPERATIONS OF $U E$

\begin{tabular}{|c|c|c|c|}
\hline Update Event & \multicolumn{4}{|c|}{ Update Operation } \\
\hline$U E_{p}$ & $o_{\text {add }}^{f_{i}}$ & $o_{\text {del }}^{f_{i}}$ & $o_{\text {mod }}^{f_{i}}$ \\
\hline$U E$ & $o_{\text {del }}^{f_{i}} \| o_{\text {mod }}^{f_{i}}$ & $o_{\text {add }}^{f_{i}}$ & $o_{\text {del }}^{f_{i}} \| o_{\text {mod }}^{f_{i}}$ \\
\hline
\end{tabular}

Specifically, the valid update operations must follow the rule shown in Table II, where $U E_{p}$ is the previous update events, and $U E$ is a new update event. For example, on the specified switch, if $U E_{p}$ involves the add operation $o_{a d d}^{f_{i}}$, the operation in $U E$ should only be $o_{d e l}^{f_{i}}$ or $o_{\text {mod }}^{f_{i}}$. Otherwise, there is another add operation for $f_{i}$ acting on the same switch, which is forbidden in the continuous update. If $U E_{p}$ involves the del operation $o_{d e l}^{f_{i}}$, the operation in $U E$ should only be $o_{a d d}^{f_{i}}$. Since once the del operation of $f_{i}$ is executed, there is no forwarding rule in the switch for $f_{i}$ that needs to be deleted or modified. Similarly, if $U E_{p}$ contains the $\bmod$ operation $o_{\text {mod }}^{f_{i}}$, the operation of $U E$ acting for the same flow on the same switch should only be $o_{d e l}^{f_{i}}$ or $o_{\text {mod }}^{f_{i}}$.

\section{Coeus Scheduling}

In this section, we will introduce modules of Coeus and present efficient scheduling algorithms to ensure the consistency policy in the continuous update. Before that, we sketch the continuous update process in Algorithm 1.

Our update process starts from a relative initial state $N S_{\text {init }}$ defined in Definition IV.1. Once an update event appears after the relative initial state, the initial operation dependency graph $G_{D}=\left(V_{D}, E_{D}\right)$ is built (lines 1-5). If the flow has existed in $N S_{\text {init }}$, we apply Algorithm 4 to divide the operation node of the flow into independent suboperation nodes to speed up the update process (lines 6,7 ). When the operation dependency graph $G_{D} \neq \emptyset$, i.e., the update events have not been finished, we check whether a new update event $N S$ arrives (lines 9). Once a new update event occurs, we split updated flows in $U E$ into existing flows and emerging flows. For existing flows, we apply Algorithm 3 and Algorithm 4 to composite the update operations and generate independent suboperation nodes in the operation dependency graph (lines 11-15). For emerging flows, we construct new operation nodes and the corresponding relationships in the operation dependency graph (lines 16-19). According to the operation dependency graph, we produce a set of update commands to update the data plane until $G_{D}=\emptyset$ (line 20).

\section{A. The Dependency Graph}

To capture the variation of link resources and the order of update operations, we construct the operation dependency graph and adjust the dependency graph dynamically to handle continuous update events. We define the operation dependency graph as follows.

Definition V.1. Operation Dependency Graph (ODG): The operation dependency graph $G_{D}=\left(V_{D}, E_{D}\right)$ is a directed graph that captures dependent relationships between update operations of each updated flow and link resources. There are two types of nodes in the ODG: the operation node $O^{f_{i}} \in V_{D}$ and the resource node $R_{u, v} \in V_{D}$. And it contains two types of edges: $\left\{R_{u, v} \leftarrow-O^{f_{i}}\right\} \in E_{D}$ and $\left\{O^{f_{i}} \leftarrow-R_{u, v}\right\} \in E_{D}$.

Specifically, the operation node $O^{f_{i}} \in V_{D}$ with a set of unexecuted update operations $\left\{o^{f_{i}}\right\}$ is labeled with the flow demand $d^{f_{i}}$. The resource node $R_{u, v} \in V_{D}$ is labeled with the residual link resource $r_{u, v}$. Initially, the link residual resource $r_{u, v}$ equals to the link capacity $c_{u, v}$. The edge $\left\{O^{f_{i}} \leftarrow--R_{u, v}\right\}$ represents that once the update operation $o^{f_{i}} \in O^{f_{i}}$ is executed, the link resource will be occupied by flow $f_{i}$. Inversely, the edge $\left\{R_{u, v} \leftarrow--O^{f_{i}}\right\}$ denotes that the update operation $o^{f_{i}} \in O^{f_{i}}$ will release the link resource. In the continuous update process, the operation dependency graph changes dynamically with update operations executed and new update events arriving.

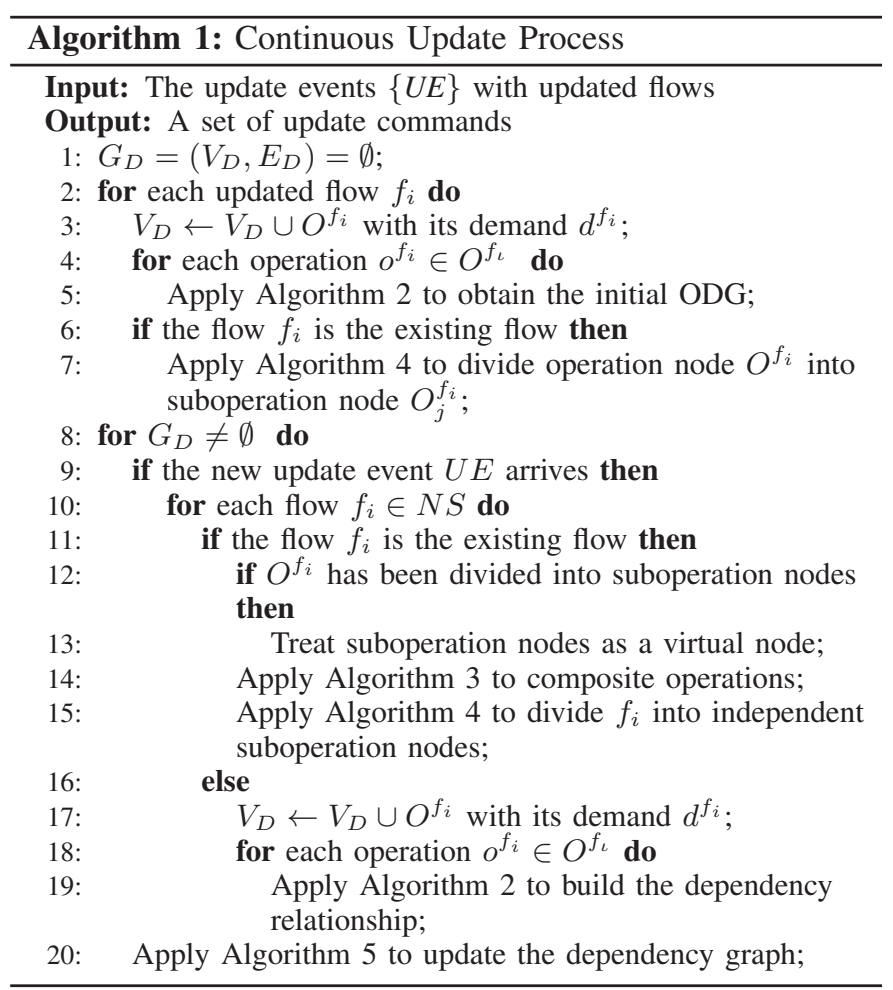

Now we discuss the construction of dependency relationship in Algorithm 2. As Algorithm 1 mentioned, we add the operation node $O^{f_{i}}$ into the ODG (lines 2, 3). Then, we judge the type of each operation $o^{f_{i}}$ for $f_{i}$ and build the corresponding dependency relationship with the resource node $R_{u, v}$ in Algorithm 2 (lines 1-13). For example, if the operation $o^{f_{i}}$ is an add operation, the forwarding rule will be added into the switch $u$ to forward $f_{i}$ to the link $\langle u, v\rangle$, and the corresponding resource of $\langle u, v\rangle$ will be consumed. We add the resource node $R_{u, v}$ and the edge $\left\{R_{u, v} \leftarrow--O^{f_{i}}\right\}$ if $R_{u, v}$ does not exist in the ODG (lines 2-4). Similarly, we build the corresponding relationship among operation nodes and resource nodes if the operation is del or mod (lines 513). Specifically, we treat a mod operation as a del operation and an add operation, and construct two directed edges $\left\{R_{u, v} \leftarrow-O^{f_{i}}\right\}$ and $\left\{O^{f_{i}} \leftarrow--R_{p, q}\right\}$ in the ODG (lines 9-13). 


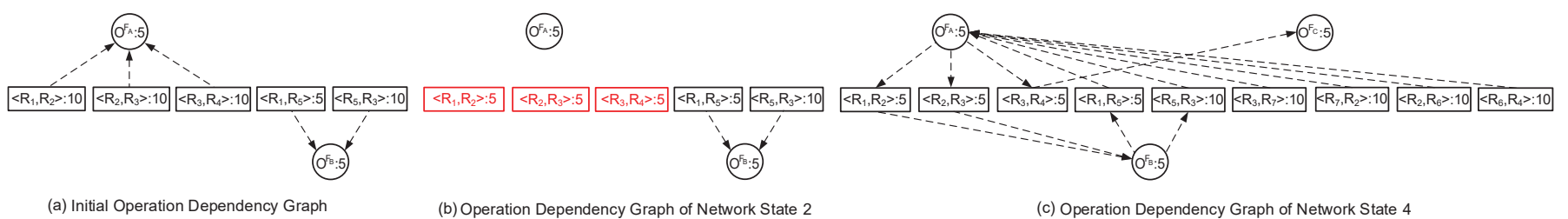

Fig. 3. The operation dependency graph of $U E_{1}, N S_{2}$, and $U E_{2}$ in Fig. 1.

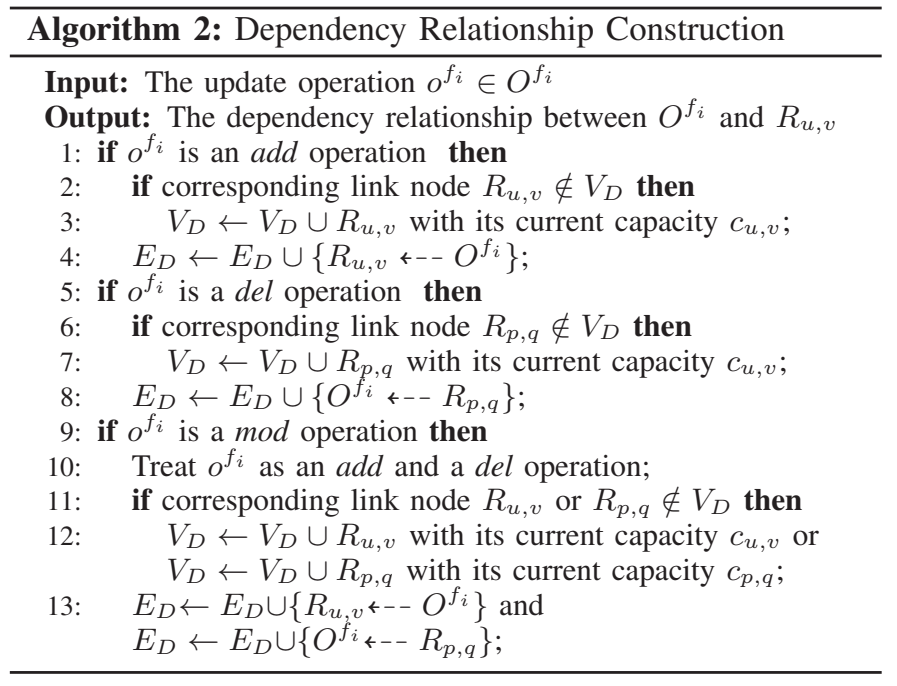

By leveraging the ODG, the state of update operations can be identified. For the unexecuted operations, the ODG maintains the relationship between the operation node and the resource node. When update operations are executed, Coeus removes update operations from operation nodes $O^{f_{i}}$ and corresponding edges between $O^{f_{i}}$ and $R_{u, v}$ in the ODG. Then, Coeus updates the link resource of $R_{u, v}$. Fig. 3 shows the ODG of $U E_{1}, N S_{2}$, and $U E_{2}$ in Fig. 1. We assume that $U E_{2}$ arises after $N S_{2}$. Fig. 3(a) corresponds to the ODG of $N S_{1}$ shown in Fig. 1(b), where the operation node $O^{f_{A}}$ contains a set of uninstalled operations $\left\{o_{\left(R_{1}, a d d\right)}^{F_{A}}\right.$, $\left.o_{\left(R_{2}, a d d\right)}^{F_{A}}, o_{\left(R_{3}, a d d\right)}^{F_{A_{A}}}\right\}$ of $F_{A}$. In $N S_{2}$ shown in Fig. 1(c), the flow $F_{A}$ has been routed on its target path, the corresponding operation node $O^{F_{A}}=\emptyset$, which is shown in Fig. 3(b). Also, corresponding directed edges are removed, and link resources are consumed. Similarly, Fig. 3(c) represents the ODG of $U E_{2}$, which means the resource request and release relationship among $F_{A}, F_{B}$, and $F_{C}$.

\section{B. Operation Composition}

Based on the ODG, we present the operation composition to reduce the number of redundant operations in the continuous update. The basic idea is that once the update event occurs, we add new update operations into existing operation nodes and construct the new dependency relationship. By checking a cycle between operation nodes and resource nodes, Coeus judges whether unnecessary operations exist and replaces redundant operations with fewer equivalent ones.

Algorithm 3 gives a detailed operation composition process. We default all of the update operations are valid. We add each valid operation $o^{f_{i}}$ into the corresponding operation node $O^{f_{i}}$ and apply Algorithm 2 to update the dependency relationship (lines 1-3). Once the new update operation $o^{f_{i}}$ incurs a cycle between $O^{f_{i}}$ and $R_{u, v}$, it is denoted that update operations $o^{f_{i}}$ and $\bar{o}^{f_{i}}$ will request and release the same link resource successively, where $\bar{o}^{f_{i}}$ is an update operation in the previous update events. We treat these operations as redundant operations. Therefore, we remove these two directed edges and corresponding update operations from the ODG (lines 4-21). Particularly, if the mod operation $o^{f_{i}}$ forms a cycle, we split $o^{f_{i}}$ as $o_{a d d}^{f_{i}}$ and $o_{d e l}^{f_{i}}$ and eliminate the redundant operations that are related to the removed edges (lines 14-20). Besides, if the residual operations of $o^{f_{i}}$ and $\bar{o}^{f_{i}}$ contain a del operation and an add operation acting on different links, we merge the residual operations into a new $\bmod$ operation $\tilde{o}^{f_{i}}$ (line 21). If $o^{f_{i}}$ does not incur a cycle while there exists another update operation $\hat{o}^{f_{i}}$ acting on the same switch, we merge these two operations into a new mod operation $\tilde{o}^{f_{i}}$ (line 23).

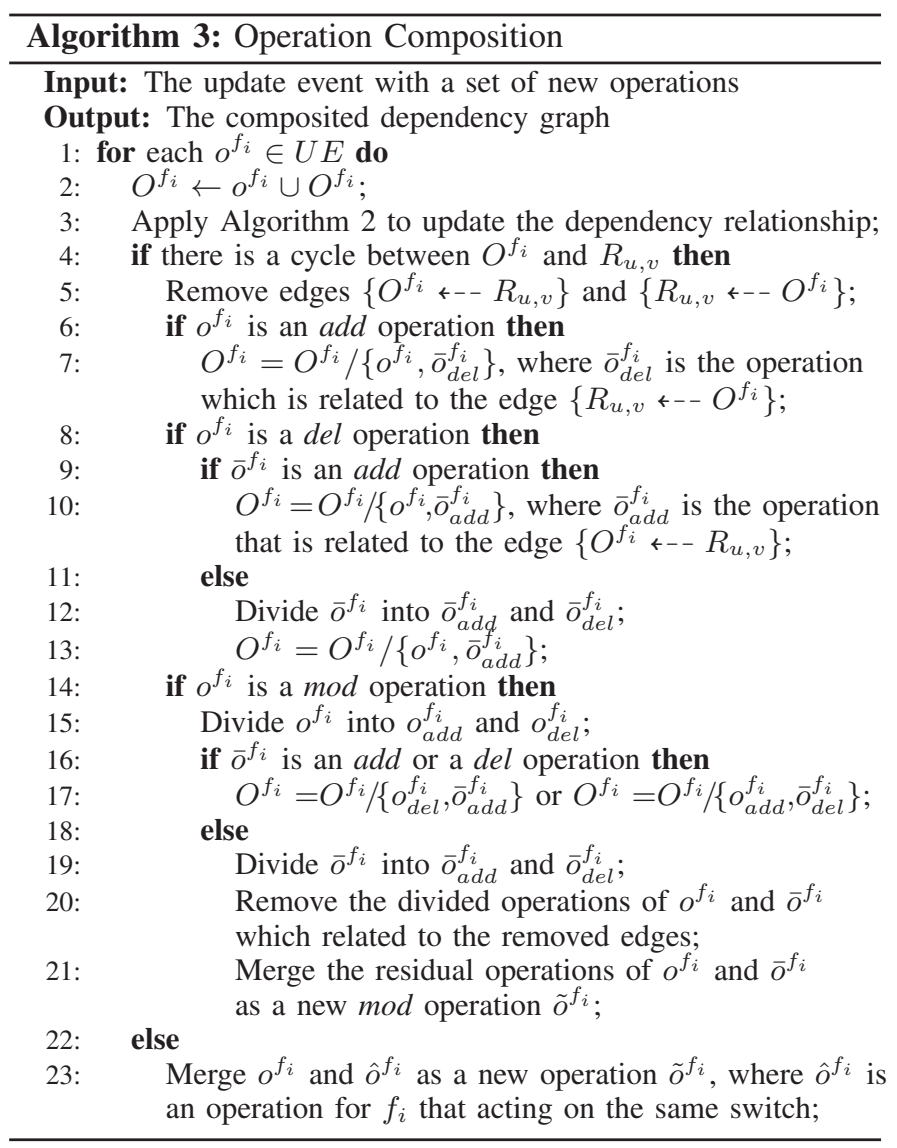

Fig. 4 is an example to illustrate the operation composition. We assume that the update event $U E_{2}$ (shown in Fig. 1(e)) oc- 


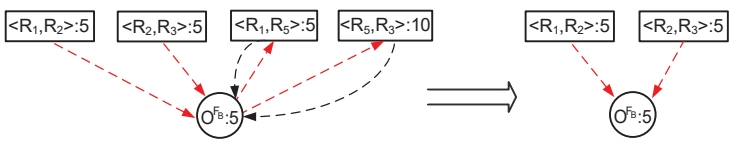

(a) Operation Composition of $N S_{2}$ and $U E_{2}$

(b) Composited Operation

Fig. 4. Illustration of update composition
TABLE III

CORRECT OPERATIONS OF SINGLE UPDATE
TABLE IV

COMPOSITION OF $U E_{m}$ AND $U E_{n}$

\begin{tabular}{|c|c|c|c|}
\hline$U E_{n} / U E_{m}$ & $o_{\text {add }}^{f_{i}}$ & $o_{\text {del }}^{f_{i}}$ & $o_{\text {mod }}^{f_{i}}$ \\
\hline$o_{\text {add }}^{f_{i}}$ & & $o_{\text {add }}^{f_{i}}$ & \\
\hline$o_{\text {del }}^{f_{i}}$ & $o_{\text {del }}^{f_{i}}$ & & $o_{\text {del }}^{f_{i}}$ \\
\hline$o_{\text {mod }}^{f_{i}}$ & $o_{\text {mod }}^{f_{i}}$ & & $o_{\text {mod }}^{f_{i}}$ \\
\hline
\end{tabular}

\begin{tabular}{|c|c|c|c|}
\hline$U E_{n} / U E_{m}$ & $o_{a d d}^{f_{i}}$ & $o_{d e l}^{f_{i}}$ & $o_{\text {mod }}^{f_{i}}$ \\
\hline$o_{a d d}^{f_{i}}$ & & $\emptyset \| \tilde{o}_{\text {mod }}^{f_{i}}$ & \\
\hline$o_{d e l}^{f_{i}}$ & $\emptyset$ & & $\tilde{o}_{\text {del }}^{f_{i}}$ \\
\hline$o_{\text {mod }}^{f_{i}}$ & $\tilde{o}_{a d d}^{f_{i}}$ & & $\tilde{o}_{\text {mod }}^{f_{i}}$ \\
\hline
\end{tabular}

curs after the network state $N S_{2}$ (shown in Fig. 1(c)). Fig. 4(a) shows the dependency relationship among the operation node $O^{F_{B}}$ and corresponding link resources under the situation mentioned above. After operation composition, the simplified dependency relationship is shown in Fig. 4(b).

Definition V.2. Correct Update Operations: The correct update operations mean that if all update operations are executed, flows are routed on their target paths, and there are no redundant forwarding rules for each flow in switches.

In the single update, all operations in the previous update events must be finished before executing the new update event. For example, we assume that two valid update events $U E_{m}, U E_{n}$ arrive successively. The update event $U E_{n}$ can be responded when all of the flows in $U E_{m}$ have been routed on their target paths. Since $U E_{m}, U E_{n}$ are valid update events, executing $U E_{m}, U E_{n}$ serially makes switches have no redundant rules. Therefore, the correct update operations can always be guaranteed in the single update. Table III represents the correct update operations in the single update, where blanks denote invalid operations. However, the single update causes lots of unnecessary operations. In Coeus, continuous update events are handled by compositing the unexecuted operations in $U E_{m}$ with the operations in $U E_{n}$. Now, we prove after operation composition, the update operations are still correct.

Theorem V.1. The graph-based operation composition produces the correct update operations.

Proof: Table IV shows the result of operation composition under the assumption that operations of $U E_{m}$ have not been executed. We prove that the final route in Table IV and Table III are equivalent. According to Algorithm 3, an add operation in $U E_{n}$ and a del operation in $U E_{m}$ may produce two types of operations after composition. One is the empty set, which means doing nothing in the specified switch. The flow still routes along its original path. This composited result corresponds to the condition where a del operation in $U E_{m}$ deletes the original route of flow, then an add operation in $U E_{n}$ adds the new route which is the same as the original route in the single update. Another possible composited result is producing a mod operation $\tilde{o}_{\text {mod }}^{f_{i}}$. The new operation $\tilde{o}_{\text {mod }}^{f_{i}}$ will delete the original route and add a new route for $f_{i}$. The flow $f_{i}$ will route along the new path. This composited result corresponds to the condition where a del operation in $U E_{m}$ deletes the original route of flow and an add operation in $U E_{n}$ adds the new route which is different from the original route in the single update. Therefore, executing the new operation produced by compositing $o_{a d d}^{f_{i}}$ in $U E_{n}$ and $o_{d e l}^{f_{i}}$ in $U E_{m}$ is equivalent to executing corresponding update operations serially in the single update. Similarly, once operations $\tilde{o}_{\text {del }}^{f_{i}}$, $\tilde{o}_{\text {add }}^{f_{i}}, \tilde{o}_{\text {mod }}^{f_{i}}$ produced by operation composition are executed, we obtain the same routes compared with the single update. We omit the proof in detail due to space constraints.

\section{Operation Node Partition}

To improve the parallelism of update, we divide the operation node into multiple suboperation nodes. We aim to generate independent suboperation nodes as many as possible while maintaining the consistency. Our algorithm is inspired by the partition technology used in [23], [24], while they either cause excessive dependencies among each partition [23] or cannot be applied to the situation where the network has potential loops [24]. In Coeus, we develop a novel partition algorithm. We prove that our algorithm is optimal to obtain independent suboperation nodes and each suboperation node can be updated in a blackhole- and loop-free manner. Now, we define suboperation node and independent suboperation node.

Definition V.3. Suboperation Node: The suboperation node $O_{j}^{f_{i}}$ owns a part of update operations of the operation node $O^{f_{i}}$. Specifically, the suboperation node contains at least one mod operation to delete the original forwarding rule and add the new forwarding rule. Besides, each suboperation node has different update operations compared with other suboperations, i.e., $O_{j}^{f_{i}} \cup O_{j+1}^{f_{i}} \cup \cdots=O^{f_{i}}, O_{j}^{f_{i}} \cap O_{j+1}^{f_{i}} \cap \cdots=\emptyset$.

In the following, we describe the process of operation node partition in Algorithm 4. $O_{j}^{f_{i}}$ is the $j$-th suboperation node of $O^{f_{i}}$. When the current operation is $o_{\text {mod }}^{f_{i}}$, we define next mod operations along the target path and the original path as $o_{(\bmod , n t)}^{f_{i}}$ and $o_{(\bmod , n o)}^{f_{i}}$ respectively. Firstly, we traverse update operations of $O_{f_{i}}$ along the target path of $f_{i}$ in reverse order (line 3). If the operation node $O^{f_{i}}$ has a mod operation $o_{\text {mod }}^{f_{i}}$, we initialize a suboperation node $O_{j}^{f_{i}}$ (lines 4,5$)$. Then we add $o_{m o d}^{f_{i}}$ into $\varphi_{o}^{f_{i}}, \varphi_{n}^{f_{i}}$ and remove $o_{m o d}^{f_{i}}$ from $O^{f_{i}}$ (lines $6,7)$, where $\varphi_{0}^{f_{i}}$ and $\varphi_{n}^{f_{i}}$ are sets of update operations acting on the original path and the target path. Since installing add operations makes $f_{i}$ routes through its target path, we add a set of add operations $\left\{o_{a d d}^{f_{i}}\right\}$ between $o_{\text {mod }}^{f_{i}}$ and $o_{(\bmod , n t)}^{f_{i}}$ into $\varphi_{n}^{f_{i}}$ (line 8). If the original path deleted by $o_{\text {mod }}^{f_{i}}$ is involved in a loop, the next suboperation node will depend on this suboperation node, i.e., the next suboperation node cannot be updated directly until this suboperation node is completed. Otherwise, a forwarding loop will occur. Next, we check whether the original path deleted by $o_{(\bmod , n o)}^{f_{i}}$ is 


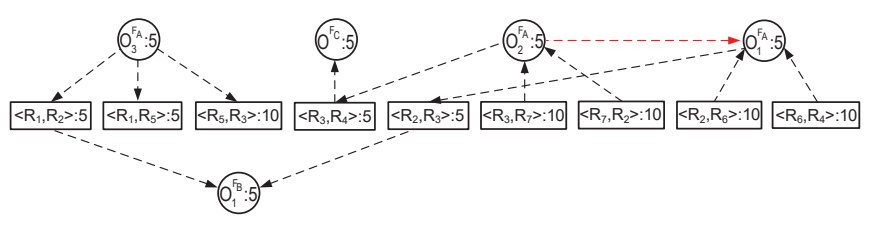

Fig. 5. Dependency graph with suboperation nodes.

involved in a loop. If this condition holds, we split $o_{(\bmod , n o)}^{f_{i}}$ into $o_{(a d d, s p)}^{f_{i}}$ and $o_{(d e l, s p)}^{f_{i}}$ and search the next mod operation $o_{(\bmod , n o)}^{f_{i}}$ along the original route of $f_{i}$ until $o_{(\bmod , n o)}^{f_{i}}$ does not incur a loop (lines 9-12). Finally, we add a set of del operation $\left\{o_{d e l}^{f_{i}}\right\}$ between $o_{\text {mod }}^{f_{i}}$ and $o_{(\bmod , n o)}^{f_{i}}$ into $\varphi_{o}^{f_{i}}$ (line 13) and construct the next suboperation node (lines 15, 16) We eliminate potential loops by this way since we add update operations which may incur loops into two suboperation nodes.

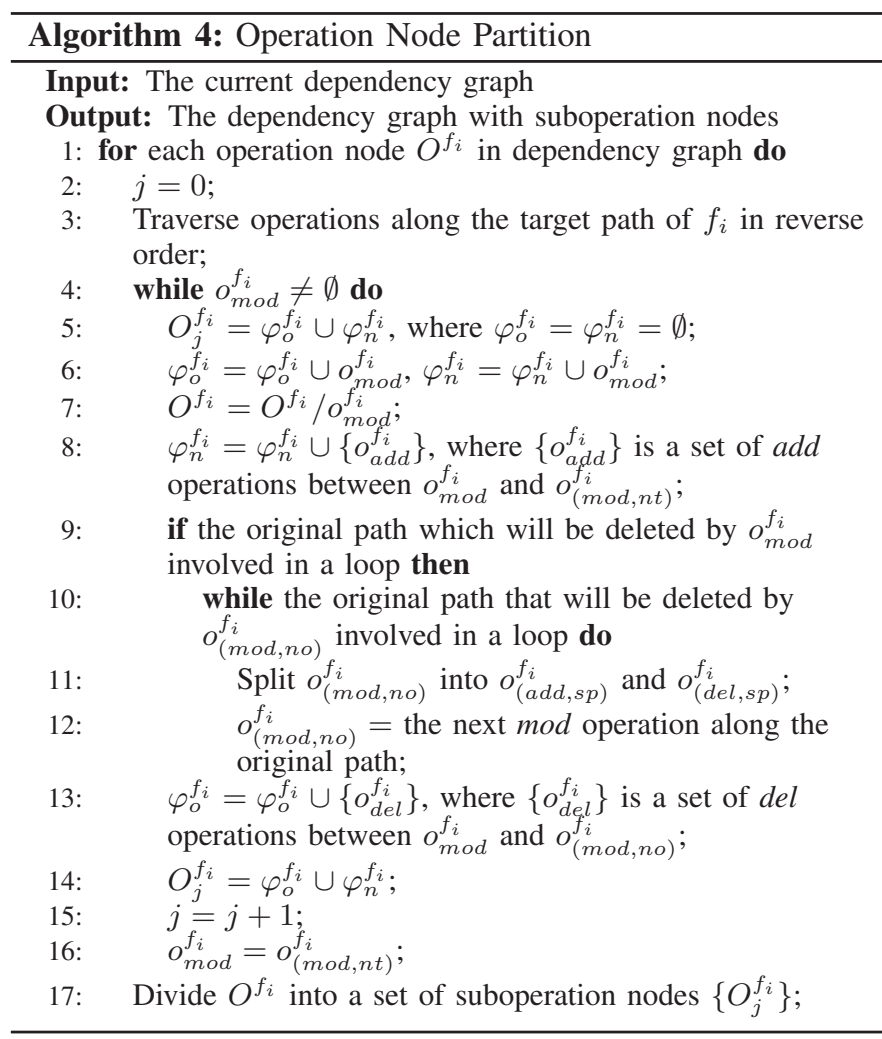

By executing Algorithm 3 and Algorithm 4, the dependency relationship among suboperation nodes is shown in Fig. 5. $O_{1}^{F_{A}}, O_{3}^{F_{A}}, O_{1}^{F_{B}}, O_{2}^{F_{B}}$, and $O_{1}^{F_{B}}$ are suboperation nodes which can be executed independently, while $O_{2}^{F_{A}}$ is a dependent node which can be executed until $O_{1}^{F_{A}}$ is completed. Now, we define the independent suboperation node formally.

Definition V.4. Independent Suboperation Node: The independent suboperation node is the suboperation node that can execute update operations independently.

Theorem V.2. The number of independent suboperation nodes obtained by Algorithm 4 is optimal.

Proof: If there is no potential loop shown in Fig.6(a), each mod operation will not incur loops. Algorithm 4 adds
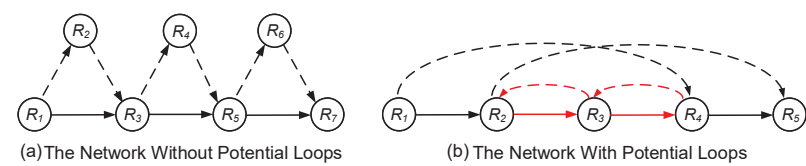

Fig. 6. Illustration of independent suboperation nodes.

each of mod operation into different suboperation nodes, i.e., the number of mod operations equals to the number of independent suboperation nodes. According to Definition V.4, Coeus produces the most number of independent suboperation nodes. If forwarding loops occur shown in Fig. 6(b), we assume that the optimal algorithm can generate an extra independent suboperation node $O_{e}^{f_{i}}$. If the original path which $O_{e}^{f_{i}}$ will be deleted is not in a potential loop, e.g., the link $\left\langle R_{1}, R_{2}\right\rangle$ or $\left\langle R_{4}, R_{5}\right\rangle$, this situation is similar to the loopfree network shown in Fig. 6(a). $O_{e}^{f_{i}}$ must be one of the independent suboperation nodes produced by Algorithm 4, which contradicts with our assumption. Otherwise, the original path which will be deleted by $O_{e}^{f_{i}}$ is involved in loops, e.g. $\left\langle R_{2}, R_{3}\right\rangle$ or $\left\langle R_{3}, R_{4}\right\rangle$. According to Definitions V.3, $O_{e}^{f_{i}}$ must has a $\bmod$ operation. And a $\bmod$ operation in $O_{e}^{f_{i}}$ acting on the target link must be involved in a loop, e.g., $o_{\left(R_{3}, \text { mod }\right)}$. Otherwise, $O_{e}^{f_{i}}$ is one of the independent suboperation nodes that we produced. $O_{e}^{f_{i}}$ cannot be an independent node under this situation because to guarantee loop-free condition, the forwarding rule that added on the target path by a mod operation in $O_{e}^{f_{i}}$ must be executed after deleting the corresponding forwarding rule of the original path.

After suboperation nodes are determined, we update each of them in the following way. Firstly, we install all add operations of the subsection node simultaneously and remove the corresponding edges and operations in the ODG. Then we install the mod operation, which makes the flow route through its target path. The residual link resources along the original path and target path are updated. Finally, we install the del operations to delete the forwarding rules acting on the original path of the flow.

Theorem V.3. The update of suboperation nodes is blackholeand loop-free.

Proof: Firstly, the update of each suboperation node requires to install the forwarding rules of the target path. Then the mod operation and del operations are executed, which ensures that packets always have the forwarding rule. Besides, each suboperation node contains the operations that will delete current forwarding rules and add target forwarding rules. Packets among suboperation nodes will not be dropped and the update is blackhole-free. In Algorithm 4, the suboperation node only contains one mod operation which is not going to form a loop. It is because that we always assign update operations incurring a potential loop to two suboperation nodes. We restrict the update order of these two suboperation nodes to ensure the loop-free condition.

\section{Update Scheduling}

After dividing the operation node into several independent suboperation nodes, the parallelism of the ODG has been 
improved. Then according to the ODG, a set of update commands are sent from the controller orderly. We describe the update scheduling in Algorithm 5.

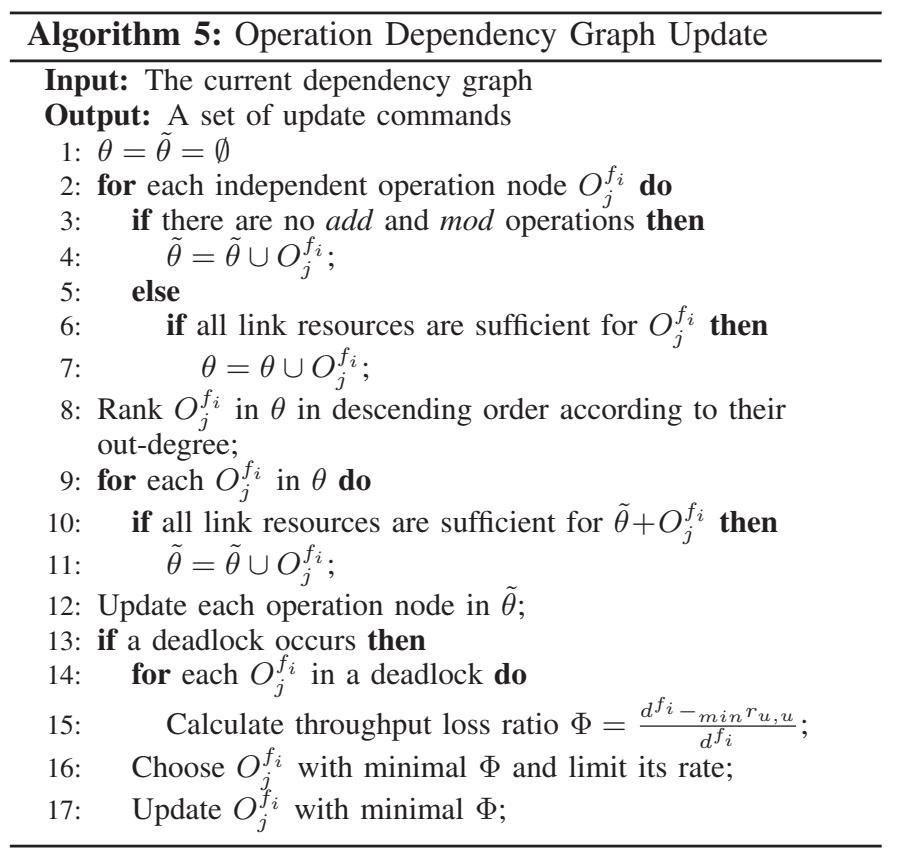

We use $\theta$ and $\tilde{\theta}$ to present the set of candidate update nodes and formal update nodes. Initially, we put the operation node $O_{j}^{f_{i}}$ which only contains del operations into $\tilde{\theta}$. Coeus can update such a $O_{j}^{f_{i}}$ directly since $O_{j}^{f_{i}}$ just releases link resources (lines 3,4$)$. Then, we find the operation nodes which have sufficient resources to update and put them into $\theta$ (lines 5-7). The candidate update nodes cannot be updated simultaneously since the residual link resources may be insufficient. To select the operation nodes which can be updated at the same time, we rank $O_{j}^{f_{i}} \in \theta$ in descending order of out-degree and add the congestion-free operations into $\tilde{\theta}$ (lines 8-11). Selecting the operation nodes with high out-degree and updating them simultaneously will release more link resources (line 8). Once deadlock occurs in the ODG, i.e., flows occupy insufficient resources of links mutually, the throughput loss ratio $\Phi$ is calculated for each operation node in deadlock. Then we select the operation node with minimal $\Phi$ and update it directly by limiting the flow rate to $d^{f_{i}}(1-\Phi)$ (lines 13-17). Once a set of update commands are sent, Coeus checks whether the new update event arises and decides the next update step.

Theorem V.4. Coeus always produces a blackhole-free, loopfree, and congestion-free update sequence.

Proof: According to TheoremV.3, suboperation nodes can be updated in a blackhole-free and loop-free manner. Besides, Algorithm 5 always updates operation nodes with sufficient link resources, which makes the network congestion-free. If deadlocks occur, we limit the rate of flow to fit the residual link resource. Therefore, updating the flow involved in the deadlock will never congest the link.

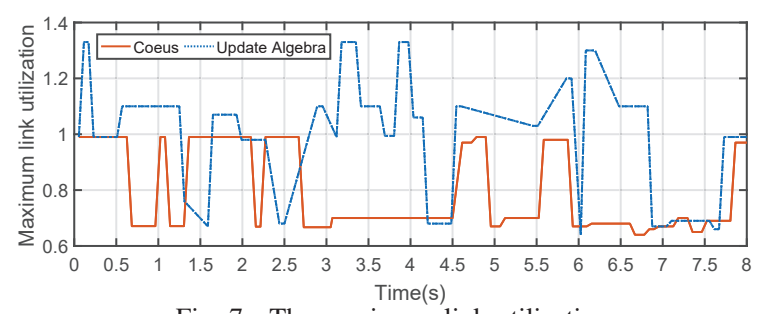

Fig. 7. The maximum link utilization.

\section{EXPERIMENTAL EVALUATION}

In this section, we design large-scale simulation experiments to verify the performance of Coeus.

Methodology: We evaluate Coeus with two common topologies: Microsoft's WAN topology [15] with 8 switches (i.e., SWAN) and 8-pods fat-tree [3] with 80 switches and 128 hosts. We set the link capacity of each topology to be 1-Gbps and then generate different numbers of updated flows (i.e., 100, $200,400,600)$ with random source and destination in the network. For each updated flow, we generate continuous update events with different arrival rate $\lambda$. Besides, according to the test of commodity switches [15], we set the time of insertion, deletion, and modification operations to be $5 \mathrm{~ms}, 5 \mathrm{~ms}, 10 \mathrm{~ms}$ respectively. The $R T T$ between the controller and switches is set to be $50 \mathrm{~ms}$. We compare the performance of Coeus with "Update Algebra" [11] which only ensures blackhole- and loop-free conditions and Cupid [23] which processes the single update with congestion-free condition guaranteed. The program executes 10 times for each data set.

Experiment results: At first, we investigate the maximum link utilization of Coeus and "Update Algebra" in Fig. 7. We do this simulation with 100 flows in SWAN topology and the arrival rate of update events $\lambda=3 / s$. Once the maximum link utilization is beyond one, the link congestion will happen. Fig. 7 shows that Coeus always guarantees that the maximum link utilization is less than or equal to one, which ensures congestion-free condition. In contrast, sometimes the maximum link utilization of "Update Algebra" is over 1.3, which incurs packets loss and degrades network performance.

Next, we generate 10 continuous update events and compare Coeus against Cupid in multiple dimensions. Fig. 8 shows the completion time of all update events. We observe that the update time of Coeus is shorter than that of Cupid. Moreover, the gap between the update time in Coeus and Cupid is getting larger with the arrival rate increasing. Specifically, in SWAN with 100 flows, Coeus shortens the makespan by $13.2 \%$, $61.4 \%, 120.7 \%, 130.1 \%, 179.8 \%$ when $\lambda=1 \sim 3$ respectively. The reason is that Cupid executes update events serially while Coeus enables the parallel execution. We also observe that the update time of Coeus in SWAN and fat-tree are highly similar. One reason is that lots of flows with sufficient resources can be updated simultaneously. Another reason is that Coeus only determines the update order of flows which may incur potential link congestion, while the number of potential congested flows in SWAN is close to that in fat-tree.

Fig. 9 shows the number of executed update operations after finishing 10 continuous update events. The number of update 


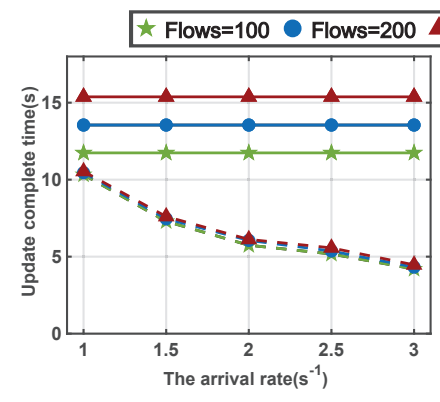

(a) WAN scenario

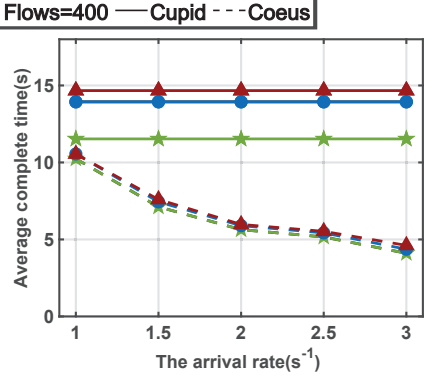

(b) Fat-tree scenario

Fig. 8. Update complete time.

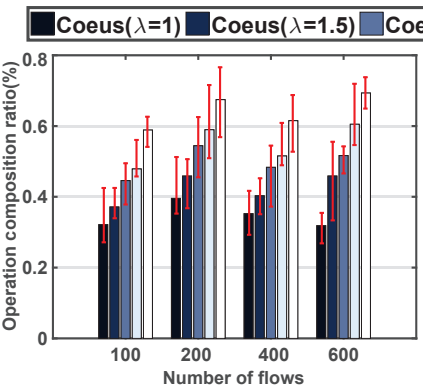

(a) WAN scenario

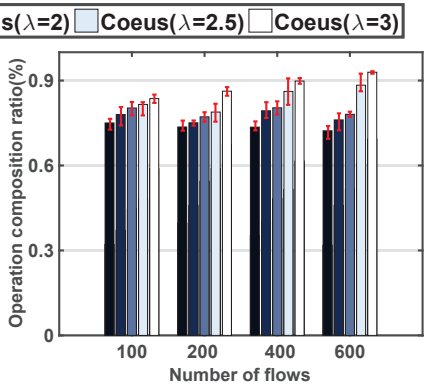

(b) Fat-tree scenario

Fig. 10. Operation composition ratio.

operations of Cupid is constant with arrival rate varying since Cupid executes all update operations for each update event. In contrast, Coeus executes fewer update operations. This benefits from the operations composition which eliminates redundant update operations. Furthermore, with the arrival ratio increasing, Coeus owns more unexecuted operations which can be composited with operations in new update events. This further reduces the number of update operations. Specifically, when the arrival rate $\lambda=3$, Coeus reduces $51.2 \%, 59.9 \%, 64.2 \%$ update operations compared with Cupid in SWAN with 100, 200, 400 updated flows. We also observe that operations in fat-tree are much more than operations in SWAN since flows in fat-tree are routed through more links. In fat-tree topology with 100, 200, 400 updated flows, when the arrival rate $\lambda=3$, Coeus reduces $82.2 \%, 83.4 \%, 90.1 \%$ operations respectively compared with Cupid since many operations are redundant in fat-tree.

Fig. 10 reflects the operation composition ratio of each update event. In SWAN, Coeus composites at least $30 \%$ redundant operations of each update event. While in fat-tree, the composition ratio is pretty high since fat-tree involves more update operations. Specifically, at least $70 \%$ operations can be composited by Coeus in each update event. This demonstrates that Coeus can execute fewer update operations while maintaining consistency in continuous updates.

\section{RELATED WORK}

With the advance of SDNs, the update problem has been widely studied. Reitblatt et al. [22] introduced the notion of consistent update in SDNs and proposed the two-phase

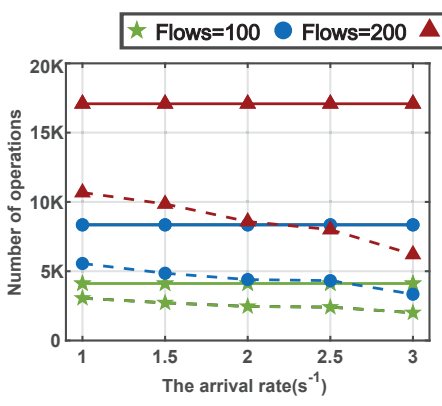

(a) WAN scenario

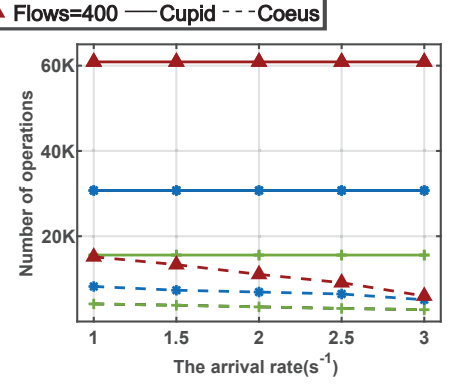

(b) Fat-tree scenario
Fig. 9. Number of operations.

update protocols to maintain per-packet coherence. To ensure the connectivity consistency, Ludwig et al [8], [17] achieved the fast blackhole- and loop-free update by using node-based protocols. To guarantee the congestion-free and fast update, zUpdate [16] and SWAN [12] utilized the slack capacity of each link to produce the static congestion-free update sequence. On these bases, Xin et al [15] and Gandhi et al. [10] performed update scheduling dynamically by utilizing the global resource dependency graph. Wang et al. [23] and $\mathrm{Wu}$ et al. [24] divided the global dependency relationship into the local dependency relationship by dividing flows into segments and updated each segment in a congestion-free manner. By taking advantage of time synchronization protocols [19]-[21], Zheng et al. [28], [30] designed heuristic algorithms to update a single flow and multiple flows at a specified time with minimum time step while maintaining the congestion-free property. Nevertheless, all of the update solutions mentioned above mainly focus on how to improve the update order of the single update. Such an update manner leads to the serial execution of continuous update events, which slows down the makespan significantly. The continuous update method is firstly proposed in [11]. The authors built the theoretical framework based on abstract algebra and generated the blackholeand loop-free update order. However, the update order in [11] may cause transient congestion which leads to packets loss and network performance degradation. To the best of our knowledge, Coeus is the first work to handle the continuous update with blackhole-, loop- and congestion-free properties guaranteed simultaneously.

\section{CONCLUSION}

We studied the consistency and continuous update problem in SDNs. We proposed Coeus to obtain a consistent update sequence during the continuous update procedure. The results of evaluation show that Coeus can reduce the makespan and redundant update operations significantly.

\section{ACKNOWLEDGMENT}

This work was supported in part by the National Key R\&D Program of China under Grant No. 2017YFB1001801, the National Natural Science Foundation of China under Grant No. 61802172, 61672276, 61872178, 61832005, 61672353, the Natural Science Foundation of Jiangsu Province under Grant No. BK20181251, and Royal Society project IEC170324. The corresponding authors are Jiaqi Zheng and Wanchun Dou. 


\section{REFERENCES}

[1] S. Agarwal, M. Kodialam, and T. Lakshman. Traffic engineering in software defined networks. In IEEE INFOCOM, pages 2211-2219, 2013.

[2] S. Akhoondian Amiri, S. Dudycz, S. Schmid, and S. Wiederrecht. Congestion-free rerouting of flows on dags. In Springer ICALP, 2018

[3] M. Al-Fares, A. Loukissas, and A. Vahdat. A scalable, commodity data center network architecture. In ACM SIGCOMM, pages 63-74, 2008.

[4] A. Basta, A. Blenk, S. Dudycz, A. Ludwig, and S. Schmid. Efficien loop-free rerouting of multiple sdn flows. IEEE/ACM Transactions on Networking, 26(2):948-961, 2018.

[5] T. Benson, A. Anand, A. Akella, and M. Zhang. Microte: Fine grained traffic engineering for data centers. In ACM CoNEXT, page 8, 2011.

[6] S. Brandt, K.-T. Förster, and R. Wattenhofer. On consistent migration of flows in sdns. In IEEE INFOCOM, pages 1-9, 2016.

[7] C.-Y. Chu, K. Xi, M. Luo, and H. J. Chao. Congestion-aware single link failure recovery in hybrid sdn networks. In IEEE INFOCOM, pages 1086-1094, 2015.

[8] K. T. Foerster, A. Ludwig, J. Marcinkowski, and S. Schmid. Loop-free route updates for software-defined networks. IEEE/ACM Transactions on Networking, PP(99):328-341, 2018.

[9] K.-T. Förster, R. Mahajan, and R. Wattenhofer. Consistent updates in software defined networks: On dependencies, loop freedom, and blackholes. In IEEE IFIP Networking, pages 1-9, 2016.

[10] R. Gandhi, O. Rottenstreich, and X. Jin. Catalyst: Unlocking the power of choice to speed up network updates. In ACM CoNEXT, pages 276282, 2017.

[11] F. L. Y.-s. L. Geng Li, Y. Richard Yang and J. Wang. Update algebra: Toward continuous, non-blocking composition of network updates in sdn. In IEEE INFOCOM, pages 1081-1089, 2019.

[12] C.-Y. Hong, S. Kandula, R. Mahajan, M. Zhang, V. Gill, M. Nanduri, and R. Wattenhofer. Achieving high utilization with software-driven wan. In ACM SIGCOMM, pages 15-26, 2013.

[13] S. Jain, A. Kumar, S. Mandal, J. Ong, L. Poutievski, A. Singh, S. Venkata, J. Wanderer, J. Zhou, M. Zhu, et al. B4: Experience with a globally-deployed software defined wan. In ACM SIGCOMM, pages 3-14, 2013.

[14] J. W. Jiang, T. Lan, S. Ha, M. Chen, and M. Chiang. Joint vm placement and routing for data center traffic engineering. In IEEE INFOCOM, pages 2876-2880, 2012.

[15] X. Jin, H. H. Liu, R. Gandhi, S. Kandula, R. Mahajan, M. Zhang, J. Rexford, and R. Wattenhofer. Dynamic scheduling of network updates. In ACM SIGCOMM, pages 539-550, 2014.

[16] H. H. Liu, X. Wu, M. Zhang, L. Yuan, R. Wattenhofer, and D. A Maltz. zupdate: updating data center networks with zero loss. In $A C M$ SIGCOMM, pages 411-422, 2013.

[17] A. Ludwig, J. Marcinkowski, and S. Schmid. Scheduling loop-free network updates: It's good to relax! In $A C M$ PODC, pages 13-22, 2015.

[18] R. Mahajan and R. Wattenhofer. On consistent updates in software defined networks. In ACM HotNets, page 20, 2013.

[19] T. Mizrahi and Y. Moses. Software defined networks: It's about time. In IEEE INFOCOM, pages 1-9, 2016.

[20] T. Mizrahi, O. Rottenstreich, and Y. Moses. Timeflip: Scheduling network updates with timestamp-based tcam ranges. In IEEE INFOCOM, pages 2551-2559, 2015.

[21] T. Mizrahi, E. Saat, and Y. Moses. Timed consistent network updates. In ACM SOSR, pages 21:1-21:14, 2015.

[22] M. Reitblatt, N. Foster, J. Rexford, C. Schlesinger, and D. Walker Abstractions for network update. In ACM SIGCOMM, pages 323-334, 2012.

[23] W. Wang, W. He, J. Su, and Y. Chen. Cupid: Congestion-free consistent data plane update in software defined networks. In IEEE INFOCOM, pages $1-9,2016$

[24] K.-R. Wu, J.-M. Liang, S.-C. Lee, and Y.-C. Tseng. Efficient and consistent flow update for software defined networks. IEEE Journal on Selected Areas in Communications, 36(3):411-421, 2018.

[25] H. Xu, Z. Yu, X.-Y. Li, L. Huang, C. Qian, T. Jung, H. Xu, Z. Yu, X.-Y. Li, L. Huang, et al. Joint route selection and update scheduling for low-latency update in sdns. IEEE/ACM Transactions on Networking, 25(5):3073-3087, 2017
[26] H. Xu, Z. Yu, X.-Y. Li, C. Qian, L. Huang, and T. Jung. Real-time update with joint optimization of route selection and update scheduling for sdns. In IEEE ICNP, pages 1-10, 2016.

[27] Z. Xu, J. Tang, J. Meng, W. Zhang, Y. Wang, C. H. Liu, and D. Yang. Experience-driven networking: A deep reinforcement learning based approach. In IEEE INFOCOM, pages 1871-1879, 2018.

[28] J. Zheng, G. Chen, S. Schmid, H. Dai, and J. Wu. Chronus: Consistent data plane updates in timed sdns. In IEEE ICDCS, pages 319-327, 2017.

[29] J. Zheng, X. Hong, X. Zhu, G. Chen, and Y. Geng. We've got you covered: Failure recovery with backup tunnels in traffic engineering. In ICNP, pages 1-10, 2016.

[30] J. Zheng, B. Li, C. Tian, K.-T. Foerster, S. Schmid, G. Chen, and J. Wu. Scheduling congestion-free updates of multiple flows with chronicle in timed sdns. In IEEE ICDCS, pages 12-21, 2018.

[31] J. Zheng, Q. Ma, C. Tian, B. Li, H. Dai, H. Xu, G. Chen, and Q. Ni. Hermes: Utility-aware network update in software-defined wans. In IEEE ICNP, pages 231-240, 2018.

[32] J. Zheng, H. Xu, G. Chen, and H. Dai. Minimizing transient congestion during network update in data centers. In IEEE ICNP, pages 1-10, 2015 .

[33] J. Zheng, H. Xu, X. Zhu, G. Chen, and Y. Geng. Sentinel: Failure recovery in centralized traffic engineering. IEEE/ACM Transactions on Networking, 27(5):1859-1872, 2019.

[34] J. Zheng, Q. Zheng, X. Gao, and G. Chen. Dynamic load balancing in hybrid switching data center networks with converters. In IEEE ICPP, pages $1-10,2019$ 